\title{
Approximate inertial manifold-based order reduction of rigid-flexible coupling manipulator
}

\author{
Lisha $\mathrm{Xu}^{1}$, Xiaoshan Qian, ${ }^{2, *}$ and Chong $\operatorname{Lin}^{1}$ \\ ${ }^{1}$ School of mechanical and electrical engineering, Central South University, Changsha 410083, China \\ ${ }^{2}$ Physical Science and Technology College, Yichun University, Yichun, Jiangxi, 336000, China
}

\begin{abstract}
An order reduction method for the flexible deformation response analysis of rigid flexible manipulators is proposed based on the approximate inertial manifold theory. This method allows a lower dimensional simplified model to be constructed from a subspace smaller than the entire state space. In this paper, truncated three-order modes are used to construct a first-order system of AIM. Compared with the traditional Galerkin method, the results show that the proposed method can reduce the degree of freedom of the system and improve the computational efficiency without obviously losing the precision of the solution, which is convenient for the subsequent vibration analysis and controller design of the system.
\end{abstract}

\section{Introduction}

Flexible manipulators have been widely used in high-tech fields. However, the dynamic characteristics of flexible manipulators are characterized by complex rigid-flexible coupling phenomenon. It is essentially a nonlinear distributed parameter system ${ }^{[1-2]}$, with infinite degrees of freedom, which brings challenges to the control of flexible manipulators to achieve accurate positioning. Therefore, the model order reduction plays an indispensable role in the control of rigid flexible manipulator. For complex nonlinear dynamic systems, the commonly used dimensionally reducing methods are traditional Galerkin method, inertial manifold method and Proper Orthogonal Decomposition (POD) method, and central manifold method ${ }^{[3]}$.In recent years, an important aspect of nonlinear dynamics has been the sensitive dependence of solutions on perturbations that may result from numerical errors and pattern truncation described above. A slight disturbance to the system can have a very important effect over a long period of time. On the other hand, for some infinite dimensional dissipative dynamic systems, asymptotically developed compact sets are called global attractors, which contain and capture nonlinear phenomena ${ }^{[4-5]}$. The inertial manifold can be used to approximate the global attractor. In practical application, the concept of Approximate Inertial Manifold (AIMS) is introduced, and AIMS is defined as a finite dimensional Lipschitz manifold and a thin surrounding neighborhood that can be entered by any orbit in a finite time. In view of this, this paper adopts the approximate inertial manifold algorithm ${ }^{[6]}$ (AIMS) to obtain the mapping relationship between the higher order

\footnotetext{
*Corresponding author: 206715@jxycu.edu.cn
} 
modes and the lower order modes, which can further reduce the order of the original system and lay a foundation for the design of the subsequent controller.

\section{Dynamic modeling of rigid flexible manipulator}

In this paper, Rigid flexible two link manipulator as the research object, the mechanical arm connected to the base of the rotating joint rigidity, flexible mechanical arm and robotic arm driven by motor shaft connection rigidity, ignore the vertical deformation of flexible manipulator, assuming that the flexible mechanical arm can be free in the plane of the bend, cross section plane after deformation and deformation after the vertical axis, as shown in figure 1 .

In Figure 1, the $\boldsymbol{O} \boldsymbol{X Y}$ coordinate system is an inertial coordinate system fixed to the base. $\boldsymbol{o}_{1} \boldsymbol{x}_{1} \boldsymbol{y}_{\mathbf{1}}$ and $\boldsymbol{o}_{2} \boldsymbol{x}_{2} \boldsymbol{y}_{2}$ are local coordinate systems fixed to the rigid manipulator and the flexible manipulator base, respectively, which will rotate with the rotation of the manipulator. $\boldsymbol{\theta}_{\mathbf{1}}$ is the rotation angle of $\boldsymbol{o}_{1} \boldsymbol{x}_{\mathbf{1}} \boldsymbol{y}_{\mathbf{1}}$ relative to $\boldsymbol{O X Y}$, and $\boldsymbol{\theta}_{\mathbf{2}}$ is the rotation angle of $\boldsymbol{o}_{2} \boldsymbol{x}_{2} \boldsymbol{y}_{\mathbf{2}}$ relative to $\boldsymbol{o}_{1} \boldsymbol{x}_{1} \boldsymbol{y}_{1}$. The length of the rigid and flexible manipulators is $\boldsymbol{l}_{\mathbf{1}}$ and $\boldsymbol{l}_{\mathbf{2}}$, respectively. According to Euler-Bernoulli beam theory, the deformation displacement of any point of the flexible manipulator is represented by a space-time function $w(x, t)$.

According to the results of reference [7], the vibration equation of the rigid-flexible coupling manipulator system can be obtained as follows:

$$
\rho_{2} \ddot{w}(x, t)+2 \delta E I_{2} \dot{w}^{(4)}(x, t)+\rho_{2} E I_{2} w^{(4)}(x, t)=F(x, t)
$$

According to the basic principle of the hypothetical mode method, the flexible body is discretized, and the deformation $\boldsymbol{w}(\boldsymbol{x}, \boldsymbol{t})$ can be expressed as:

$$
w=\sum_{i=1}^{N} \Phi_{i}(x) \cdot q_{i}(t)
$$

Where, $\mathrm{N}$ is the order of modes, $\Phi_{i}(x)$ is the $\mathrm{i}$-th mode shapes, and $q_{i}(t)$ is the corresponding mode coordinates.

According to the boundary conditions of the cantilever beam, the modal shape function of the flexible arm can be expressed as:

$$
\Phi_{i}(x)=\cosh \gamma_{i} x-\cos \gamma_{i} x-k_{i}\left(\sinh \gamma_{i} x-\sin \gamma_{i} x\right)
$$

In the formula, $\gamma_{i}$ is the eigenvalue, and $i$ is the mode order.

The solution of Equation (1) is projected onto the orthogonal basis of space, and the approximate solution of Galerkin process is obtained[8], i.e

$$
z=\sum_{i=1}^{2 N} \ddot{q}_{i}(t) \cdot \Phi_{i}(x)+E I_{2} \sum_{i=1}^{2 N} q_{i}(t) \cdot \frac{d^{4} \Phi_{i}(x)}{d x^{4}}+\frac{2 \delta E I_{2}}{\rho_{2}} \sum_{i=1}^{2 N} \dot{q}_{i}(t) \frac{d^{4} \Phi_{i}(x)}{d x^{4}}-f(x, t)
$$

For the convenience of analysis, $S_{k}$ is used here to represent the orthogonal projection in the Hilbert space $\mathrm{H}$ that will be covered by the first k eigenfunctions of A. $S_{k} H$ and $G_{k} H$ are called low and high mode subspaces respectively. In this way, $G_{k}$ is projected onto an infinite dimensional space, so it has to be truncated. Define $S=S_{k} w$ and $G=G_{k} w$ and apply them to Equation (4).A set of equations of the following form can be obtained[9]:

$$
\ddot{S}+A_{1}(\dot{S}+\dot{G}, \dot{S}+\dot{G})+B_{1}(S)=f_{1}
$$




$$
\ddot{S}+A_{2}(\dot{S}+\dot{G}, \dot{S}+\dot{G})+B_{2}(G)=f_{2}
$$

Where, $A_{1}(\dot{S}+\dot{G}, \dot{S}+\dot{G}), A_{2}(\dot{S}+\dot{G}, \dot{S}+\dot{G})$ is a nonlinear term.

Clearly, the traditional Galerkin method was obtained by setting $\dot{G}=0$ in Equation (6), ignoring the interaction between low and high modes.However, according to the approximate inertial manifold theory, an approximate inertial manifold[10] can be constructed as follows:

$$
A_{2}(\dot{S}, \dot{S})+B_{2}(G)=f_{2}
$$

That is $G=\Psi(S)$, capturing high patterns with low patterns. Therefore, the solution of Equation (2) can be expressed as:

$$
w(x, t)=S+\Psi(G(t))
$$

Where $q_{k}(t)=\left(f(x, t), \Phi_{k}\right) /\left(\Phi i H_{i}^{4}+E I_{2}\right), i=1,2, \ldots, 2 N, k=N+1, N+2, \ldots, 2 N$

$$
f(x, t)=F(x, t) / \rho_{2}
$$

According to the results of model validation in this reference [7], the infinite dimensional discrete system of the flexible manipulator can be approximated by the first three order models.From Equation 1 and Equation 7, we can get:

$$
\begin{aligned}
& q_{3}=\tau_{2} m_{33}-\left\{\left[2 m_{33} \rho_{2} L_{2}\left(\dot{\theta}_{1}+\dot{\theta}_{2}\right) q_{1}-k_{3} m_{23}\right] \dot{q}_{1}+\rho_{2} L_{1} \cos \theta_{2} \cdot h_{1} \dot{\theta}_{1}^{2} m_{33}-\left(\delta_{3}-\right.\right. \\
& \left.\left.\rho_{2}\left(\dot{\theta}_{1}+\dot{\theta}_{2}\right)^{2} m_{23}\right) q_{1}\right\}+\rho_{2} L_{1} \cos \theta_{2} \cdot h_{2} \dot{\theta}_{1}^{2} m_{33} \cdot \rho_{2} L_{2} \dot{\theta}_{1}^{2} \sin \theta_{2} \cdot h_{2} /\left(\rho_{2}\left(\dot{\theta}_{1}+\dot{\theta}_{2}\right)^{2}-\delta_{4}\right) \\
& +\left[k_{2} \dot{\theta}_{2}+\delta_{2} \theta_{2}+\frac{1}{2} \rho_{2} L_{1} L_{2}^{2} \sin \theta_{2} \cdot \dot{\theta}_{1}^{2}\right] m_{33}-\rho_{2} L_{2} \dot{\theta}_{1}^{2} \sin \theta_{2} \cdot h_{1} m_{23} / \rho_{2} L_{1} \cos \theta_{2} \cdot h_{3} \dot{\theta}_{1}^{2} m_{33} \\
& q_{2}=\tau_{2} m_{33}-\left\{\left[2 m_{33} \rho_{2} L_{2}\left(\dot{\theta}_{1}+\dot{\theta}_{2}\right) q_{1}-k_{3} m_{23}\right] \dot{q}_{1}+\rho_{2} L_{1} \cos \theta_{2} \cdot h_{1} \dot{\theta}_{1}^{2} m_{33}-\left(\delta_{3}-\right.\right. \\
& \left.\left.\rho_{2}\left(\dot{\theta}_{1}+\dot{\theta}_{2}\right)^{2} m_{23}\right) q_{1}\right\}+\rho_{2} L_{1} \cos \theta_{2} \cdot h_{3} \dot{\theta}_{1}^{2} m_{33} \cdot \rho_{2} L_{2} \dot{\theta}_{1} \sin \theta_{2} \cdot h_{3} /\left(\rho_{2}\left(\dot{\theta}_{1}+\dot{\theta}_{2}\right)^{2}-\delta_{5}\right) \\
& +\left[k_{2} \dot{\theta}_{2}+\delta_{2} \theta_{2}+\frac{1}{2} \rho_{2} L_{1} L_{2}^{2} \sin \theta_{2} \cdot \dot{\theta}_{1}^{2}\right] m_{33}-\rho_{2} L_{2} \dot{\theta}_{1}^{2} \sin \theta_{2} \cdot h_{1} m_{23} / \rho_{2} L_{1} \cos \theta_{2} \cdot h_{2} \dot{\theta}_{1}^{2} m_{33}
\end{aligned}
$$

Where, $m_{23}=\rho_{2} \int_{0}^{l_{2}} x \cdot \Phi_{1}(x) d x \quad m_{33}=\rho_{2} \int_{0}^{l_{2}} \Phi_{1}(x) d x$

Finally, the obtained $q_{2}$ and $q_{3}$ can be substituted into the following vibration displacement equation to obtain the first-order vibration model of the flexible manipulator:

$$
w(x, t)=\Phi_{1}(x) q_{1}+\Phi_{2}(x) q_{2}+\Phi_{3}(x) q_{3}
$$

\section{Simulation analysis and experimental verification}

\subsection{Simulation Analysis}


The initial state of the rigid and flexible manipulators are assumed in a horizontal position; that is, $\theta_{1}=\theta_{2}=q_{1}=q_{2}=q_{3}=0$. The joint drive torque is $\tau_{1}=\tau_{2}=0.412 \mathrm{~N} / \mathrm{m}$. The size of the flexible manipulator is $300 \mathrm{~mm} \times 20 \mathrm{~mm} \times 15 \mathrm{~mm}$. The other physical parameters of the system are shown in Table 1.The calculation results of the traditional Galerkin method (TGM) and the proposed order reduction method based on approximate inertial manifold (AIM) are compared under different mode numbers.Calculate the time step and take the length as $1 \times 10^{-2} s$, and calculate the time step altogether $2 \times 10^{3}$.Fig. 2 shows the time history of terminal vibration displacement at $x=0.3$ in different modes.It can be seen from the figure that the end of the flexible arm goes through similar attenuating oscillations and finally converges to the same equilibrium point, and the system oscillates from the initial state $_{w=0}$ and finally returns to the initial state.By comparing Fig. 1(a) and Fig. 1(b), it can be seen that the TGM method and AIM method are in good agreement with each other for different mode numbers, which shows that the proposed method can accurately describe the transient and steady-state behavior of the system with the accuracy of the traditional method.

Table 1. System parameters of the rigid-flexible manipulator.

\begin{tabular}{|l|l|l|}
\hline Physical parameters & Rigid manipulator & Flexible manipulator \\
\hline Length $/ \mathrm{m}$ & $\mathrm{L}_{1}=0.33$ & $\mathrm{~L}_{1}=0.30$ \\
\hline Moment of inertia $/ \mathrm{kg} \cdot \mathrm{m}^{2}$ & $\mathrm{~J}_{1}=0.0812$ & $\mathrm{~J}_{2}=0.138$ \\
\hline Linear density $/ \mathrm{kg} \cdot \mathrm{m}^{3}$ & & $\rho_{2}=0.4865$ \\
\hline Elastic modulus $/ \mathrm{N} \cdot \mathrm{m}^{2}$ & & $\mathrm{EI}_{2}=26.055$ \\
\hline Mass of the end $/ \mathrm{kg}$ & $\mathrm{M}_{\mathrm{t}}=0.721$ & $\mathrm{M}_{\mathrm{p}}=0.5$ \\
\hline
\end{tabular}

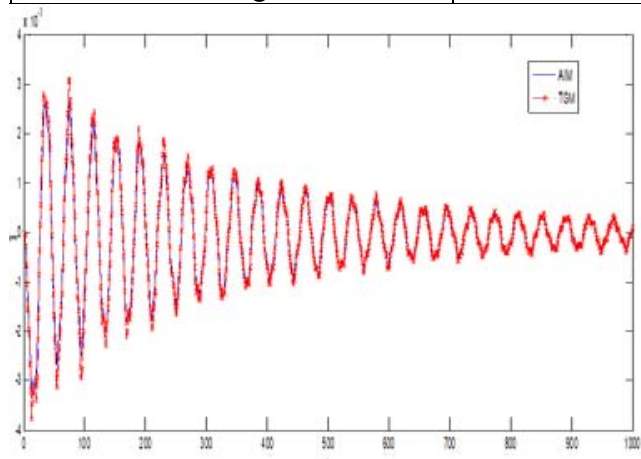

(a) Modes are 5

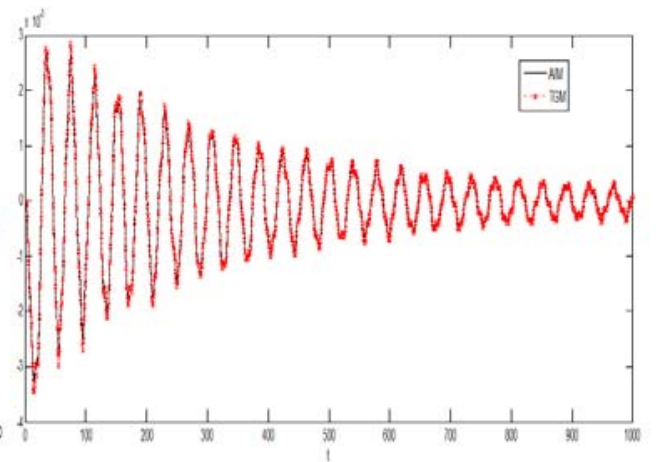

(b) Modes are 10

Fig.1. Vibration displacement in different modes.

\subsection{Experimental Verification}

The initial state of the rigid and flexible manipulators are assumed in a horizontal position; that is, The joint drive torque is . The size of the flexible manipulator is The other physical parameters of the system are shown in Table 1.

The calculation results of the traditional Galerkin method (TGM) and the proposed order reduction method based on approximate inertial manifold (AIM) are compared under different mode numbers.Calculate the time step and take the length as, and calculate the time step altogether .Fig. 2 shows the time history of terminal vibration displacement at $\mathrm{x}=0.3$ in different modes.It can be seen from the figure that the end of the flexible arm goes through similar attenuating oscillations and finally converges to the same equilibrium point, and the system oscillates from the initial state and finally returns to the initial state.By 
comparing Fig. 2(a) and Fig. 2(b), it can be seen that the TGM method and AIM method are in good agreement with each other for different mode numbers, which shows that the proposed method can accurately describe the transient and steady-state behavior of the system with the accuracy of the traditional method.

In order to further verify the effectiveness of the dynamic model and dimension reduction method, developed an experimental platform for the coupled mechanical arm, sensor device is composed of two encoders and three strain gauge, for measuring the different position of the deformation, strain measurement using arms half bridge circuit connection, the measured voltage signal, through the strain amplifier instrument amplifier, again by the acquisition of high speed data acquisition instrument and A/D conversion, and through the computer to calculate the end point of the deformation, as shown in figure 2 .

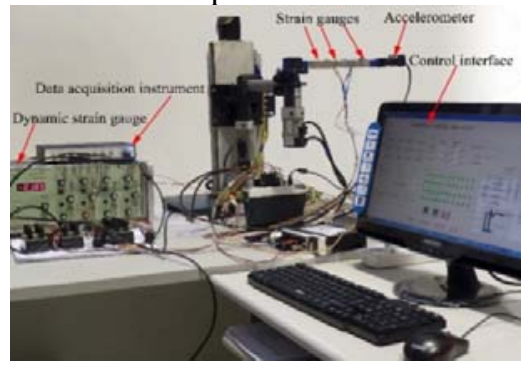

Fig.2. Rigid-flexible manipulator experimental platform.

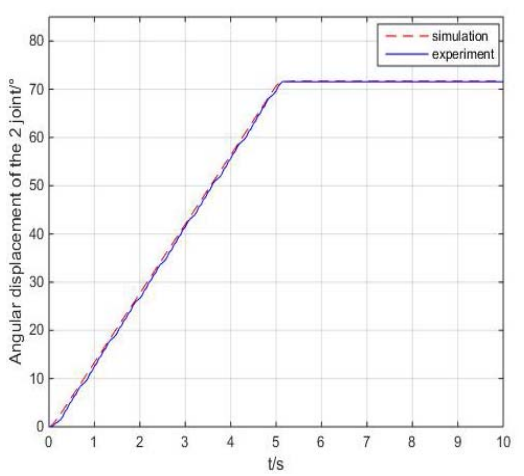

(a) Flexible joint angular displacement

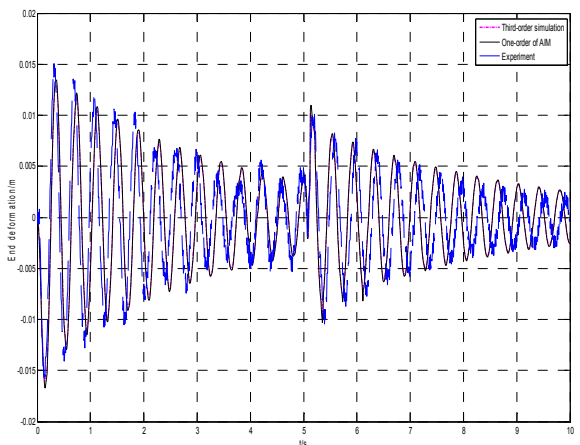

(b) Deformation of flexible joint end

Fig. 3. Comparison of simulation results with experimental results.

Within $5 \mathrm{~s}$ by controlling the card to the stepper motor a voltage signal, $5 \mathrm{~s}$ retreat to voltage signal, through the motor encoder to get results of angular displacement is calculated from the voltage value of the strain gauge at the end of the deformation, the dimension reduction after comparing the results with the experimental results of the model, as shown in figure 4, from the picture can draw the following conclusion:

(1) It can be seen from Fig. 3(a) that the simulation results of the angular displacement of the flexible joint are basically consistent with the experimental results, which proves that the spectral method and Galerkin truncation theory can accurately describe the dynamic characteristics of the flexible manipulator.

(2) As can be seen from the figure 3 (b) the AIM approximation of the first-order system after the simulation results and the simulation results of the three order system, and within $5 \mathrm{~s}$ before two kinds of simulation result was consistent with the experimental results, voltage signal, In the fifth second, the end deformation of the flexible arm changes abruptly, finally in the structure under the action of damping gradually eliminate, proved with the 
AIM to further reduce the effectiveness of the existing three order system, laid a theoretical foundation for the follow-up control study.

\section{Conclusion}

The rigid-flexible coupling manipulator equations based on Lagrange method are simplified by approximate inertial manifold method.Compared with the traditional Galerkin method, the proposed method takes into account the interaction between the lower and higher modes, reduces the computation time, and improves the distance between the original system and the simplified system in the long-term behavior.In addition, the simplified first order approximate inertial popular model lays a theoretical foundation for the design of subsequent controllers.

\section{Acknowledgments}

This work was financially supported by the National Natural Science Foundation of China (61963036), the Jiangxi Provincial Department of Education Science and Technology Project (GJJ201610).

\section{References}

1. S. K. Dwivedy, Eberhard P, Dynamic analysis of flexible manipulators, a literature review [J]. Mechanism and machine theory, 2006,41(7):749-777.

2. Z. Tiemin, G. Zhi, Model reduction of flexible manipulators[C]//Proceedings of the 3rd World Congress on Intelligent Control and Automation, IEEE,2000,1:95-98.

3. O. Ouwole, P. I. Barton, W. H. Green, Obtaining accurate solutions using reduced chemical kinetic models: a new model reduction method for models rigorously validated over ranges[J]. Combustion Theory and Modelling, 2007,11(1):127-146.

4. J. Z. Zhang, Y. Liu, P. H. Feng. Approximate inertial manifolds of Burgers equation approached by nonlinear Galerkin's procedure and its application[J]. Communications in Nonlinear Science and Numerical Simulation,2011,16(12):4666-4670.

5. B. J. Gross, P. J. Atzberger, Hydrodynamic flows on curved surfaces: Spectral numerical methods for radial manifold shapes [J].Journal of Computational Physics, 2018,115(10):663-689.

6. X.H. Li, Inertial manifolds for the 3D modified-Leray- a model [J]. Journal of Differential Equations, 2020,268(4):1532-1569

7. Y. Pan, Research on dimension reduction and control of rigid flexible manipulator model based on spectrum method[D]. Changsha: Central South University. 2011.

8. W. Kang, J.Z. Zhang, S.Ren, et al. Nonlinear Galerkin method for low-dimensional modeling of fluid dynamic system using POD modes [J]. Commun Nonlinear Sci. 2015,22(1):943-952.

9. A.M. Maryam, Hassanaly, V. Raman, A priori analysis of reduced description of dynamical systemsusing approximate inertial manifolds [C]. Commun Nonlinear Sci. 2020,409(2):1-20.

10. J.Z. Zhang, S. Ren, G.H. Mei, Model reduction on inertial manifolds for $\mathrm{n}-\mathrm{s}$ equations approached by multilevel finite element method. Commun Nonlinear Sci. 2011,16:195205. 